\title{
Stabilizing an attractive Bose-Einstein condensate by driving a surface collective mode
}

\author{
Arjendu K. Pattanayak, Arnaldo Gammal, ${ }^{*}$ Charles A. Sackett, ${ }^{\dagger}$ and Randall G. Hulet \\ Department of Physics and Rice Quantum Institute, Rice University, Houston, Texas 77251-1892
}

(Received 8 June 2000; published 6 February 2001)

\begin{abstract}
Bose-Einstein condensates with attractive interatomic interactions undergo collective collapse beyond a critical number. We show theoretically that if the low-lying collective modes of the condensate are excited, the radial breathing mode further destabilizes the condensate. Remarkably, excitation of the quadrupolar surface mode causes the condensate to become more stable, imparting quasiangular momentum to it. A significantly larger number of atoms may then occupy the condensate. Efforts are under way for the experimental realization of these effects.
\end{abstract}

DOI: 10.1103/PhysRevA.63.033604

PACS number(s): 03.75.Fi, 32.80.Pj, 05.30.Jp, 67.40.Db

Bose-Einstein condensation in cold and dilute atomic gases [1] provides a domain for studying nonlinear quantum systems [2]. The condensates with attractive interatomic interactions are particularly remarkable. These were initially argued to be impossible [3], but it is now established that as long as the number of condensate atoms $N_{0}$ is less than a maximum $N_{\mathrm{s}}$ [4], an attractive condensate may exist in a metastable ground state. As such, the nonlinear interatomic interactions not only alter the properties of such condensates from those of an ideal gas but also entirely govern its fate. This is best understood in the mean-field view, where the relevant elements are the zero-point kinetic energy of the atoms, the confining harmonic trap potential $V(\vec{r})$ and the interatomic interactions scaling as $a N_{0}|\psi|^{2}$, where $a$ is the $s$-wave scattering length for the gas. The condensate is then described by the product wave function $\phi=\sqrt{N_{0}} \psi$ where the Gross-Pitaevskii (GP) equation [2]

$i \hbar \frac{\partial}{\partial t} \psi(\vec{r}, t)=\left[-\frac{\hbar^{2}}{2 m} \nabla^{2}+V(\vec{r})+\frac{4 \pi \hbar^{2} a N_{0}}{m}|\psi(\vec{r})|^{2}\right] \psi(\vec{r}, t)$

governs the single-particle wave function $\psi$ of the weakly interacting Bose condensate at zero temperature. A condensate wave function exists as long as the outward pressure of the kinetic energy term balances the squeezing tendency of the other two terms, so that $N_{0}$ must be less than a maximum $N_{\mathrm{s}}$ [4]. Beyond this number, the wave function "collapses," tending to the singular limit of a point object. In physical condensates, this is halted by dissipation: The collapse rapidly increases the density of the condensate and as a result the rates for inelastic processes, including two-body dipolar collisions and three-body recombination, are sharply enhanced. The atoms participating in these processes acquire large kinetic energies and are ejected from the condensate. This depletion of $N_{0}$ (the "burning off'" of atoms) decreases the nonlinearity thus slowing and finally halting the collapse. The initial collapse can be triggered by thermal and quantal

\footnotetext{
*Present address: Instituto de Física Teórica, Universidade Estadual Paulista, 01405-900 São Paulo, Brazil.

†Present address: Time and Frequency Division, National Institute of Standards and Technology, Boulder, CO 80303.
}

fluctuations and may also involve macroscopic quantum tunneling. Until recently, this process was obtained only in the Rice ${ }^{7} \mathrm{Li}$ experiments $[5,6]$. Theoretical descriptions are thus far incomplete $[7,8]$.

After these pioneering observations, attention has now turned to the control of this unusual quantum phenomenon. A new experiment at JILA [9] has since been able to manipulate the collapse in a ${ }^{85} \mathrm{Rb}$ condensate by changing the scattering length of the atoms using a Feschbach resonance in a strong magnetic field. These experiments start with a nearly pure condensate in approximate equilibrium (zero fillrate) with a large condensate number $N_{0}$ and a positive scattering length. The magnetic field is then changed so that the scattering length becomes negative. Since $N_{0} \gg N_{\mathrm{s}}$, the condensate is out of equilibrium and goes through non-trivial dynamics, burning off a significant fraction of the condensate in the process. The scattering length is then made positive again so that the condensate can be imaged and analyzed.

These experiments, which are not yet completely understood, indicate a connection between the dynamics and kinetics of attractive Bose condensates. In this paper, we clarify this connection and show theoretically that the collapse kinetics are strongly affected by a weak perturbation of the harmonic potential within which the condensate is trapped. We have considered general potentials $V(\vec{r})$ $=\frac{1}{2} m \sum_{i=1}^{3} \omega_{i}^{2} x_{i}^{2}$ where $\vec{r} \equiv\left(x_{1}, x_{2}, x_{3}\right) \equiv(x, y, z)$ and the parameters $\omega_{i}$ characterize the trapping potential along the three axes. For the case presented in detail below, $\omega_{i}^{2}$ $=\omega_{0}^{2}\left[1+\alpha_{i} \cos (\omega t)\right]$ where $\omega$ is the frequency of the forces and the $\alpha_{i}$ are its amplitudes. This corresponds to a spherically symmetric trap driven asymmetrically by a weak, sinusoidally time-dependent field. We have been able to obtain results from an exact numerical simulation of the nonlinear Schrödinger equation in cylindrical coordinates. The best physical insight into the results are obtained from a Gaussian time-dependent variational principle approximation [7] (GVA) analysis of the GP equation, however, and we shall emphasize this in the following. The Gaussian ansatz is motivated by the shape of the ground state for noninteracting particles in a harmonic trap and constrains the condensate wavefunction to be of the form $\psi(\vec{r}, t)=\psi_{x} \psi_{y} \psi_{z}$ where $\psi_{i}$ $=\left[2 \pi a_{i}(t)\right]^{-1 / 4} \exp \left\{-x_{i}^{2}\left[1 / 4 a_{i}(t)+i b_{i}(t)\right]\right\}$. This ansatz when used in Eq. (1) yields that the condensate dynamics 
correspond [10] to a classical effective Hamiltonian. With the transformation [11] $a_{i}(t)=\rho_{i}^{2}(t)$ and $b_{i}(t)$ $=\Pi_{i}(t) / 2 \rho_{i}(t)$ this Hamiltonian has the transparent form

$$
H_{v a r}=\sum_{i}\left[\frac{\Pi_{i}^{2}}{2 m}+\frac{\hbar^{2}}{8 m \rho_{i}^{2}}+\frac{m \omega_{i}^{2} \rho_{i}^{2}}{2}\right]+\frac{\hbar^{2} a N_{0}}{4 \sqrt{\pi}} \frac{1}{\rho_{1} \rho_{2} \rho_{3}} .
$$

The parameters $\rho_{i}$ and $\Pi_{i}$ are given by $\left\langle\hat{x}_{i}^{2}\right\rangle=\rho_{i}^{2}$ and $\left\langle\hat{x}_{i} \hat{p}_{i}\right.$ $\left.+\hat{p}_{i} \hat{x}_{i}\right\rangle=2 \rho_{i} \Pi_{i}$. Note that $\left\langle\hat{x}_{i}\right\rangle=0=\left\langle\hat{p}_{i}\right\rangle$ for this wave function. The centroid of the condensate thus sits at the trap minimum and all the dynamics is restricted to changes in the condensate width $\rho_{i}$ in the three directions. That is, the dynamics of a quasiparticle with canonically conjugate position and momentum variables $\rho_{i}$ and $\Pi_{i}$ characterizes the condensate. A further scaling by the length of the trap $\ell_{0}$ $=\sqrt{\hbar /\left(m \omega_{0}\right)}$, the time scale $\omega_{0}^{-1}$, and energy $\hbar \omega_{0}$ yields the dimensionless Hamiltonian

$$
H_{v a r}=\frac{1}{4} \sum_{i}\left[\frac{\Pi_{i}^{2}}{2}+\frac{1}{\rho_{i}^{2}}+\left(1+\alpha_{i} \cos (\omega t)\right) \rho_{i}^{2}\right]-\frac{3 \beta}{4 \rho_{1} \rho_{2} \rho_{3}},
$$

where $\beta=[4 /(3 \sqrt{2 \pi})]\left(N_{0}|a| / \ell_{0}\right)$. The kinetics of the condensate, including the fill rate and dissipative losses, affect the dynamics through the time dependence of $N_{0}$. We set the fill rate $G_{0}$ to a constant [12], which is a good approximation to the results from the Boltzmann equation [7] over the time scales considered. Further, dipolar relaxation scales as $\phi^{2}$, while molecular recombination is a three-body process scaling as $\phi^{3}$. In particular, with the Gaussian approximation for the wave function, we can write

$$
\dot{N}_{0}=G_{0}-\frac{N_{0}^{2}}{\pi^{3 / 2} \rho_{1} \rho_{2} \rho_{3}}\left(\frac{G_{2}}{2 \sqrt{2}}+\frac{N_{0} G_{3}}{6 \sqrt{3} \pi^{3 / 2} \rho_{1} \rho_{2} \rho_{3}}\right),
$$

where $G_{1}$ and $G_{2}$ are the appropriate rate constants [13]. The Hamiltonian equation (3) and the kinetics equation (4) define the condensate dynamics completely.

Consider the fixed points of the Hamiltonian given by $\partial H_{v a r} / \partial \Pi_{i}=0=\partial H_{v a r} / \partial \rho_{i}$, which yield $\Pi_{i}=0$ and a nonzero $\rho_{i}$ corresponding to the width of a metastable condensate. This solution $\rho_{\beta} \equiv\left|\vec{\rho}_{\beta}\right|$ is strongly affected by the attractive hole at the origin $(\vec{\rho}=0$ or infinite condensate density) due to the term $\beta /\left(\rho_{1} \rho_{2} \rho_{3}\right)$ in the Hamiltonian. The condensate is only stable when the quasiparticle avoids this attractive hole. As $N_{0}$ and correspondingly $\beta$ increase, the metastable minimum moves closer to the origin, becomes shallower until it becomes an inflection point, and finally vanishes. Correspondingly, the width $\rho_{\beta}$ decreases abruptly when the minimum vanishes, leading to a collapse via the kinetics. The critical values $\rho_{m}$ and $\beta_{m}$ for collapse are determined by further imposing the inflection condition $d^{2} H_{v a r} / d \rho_{i}^{2}=0$. This gives $\rho_{m}=5^{-1 / 4}$ and $\beta_{m}=\frac{8}{3} 5^{-5 / 4}$, corresponding to $N_{\mathrm{s}} \approx 1400$ atoms for the conditions of the Rice experiment [5].

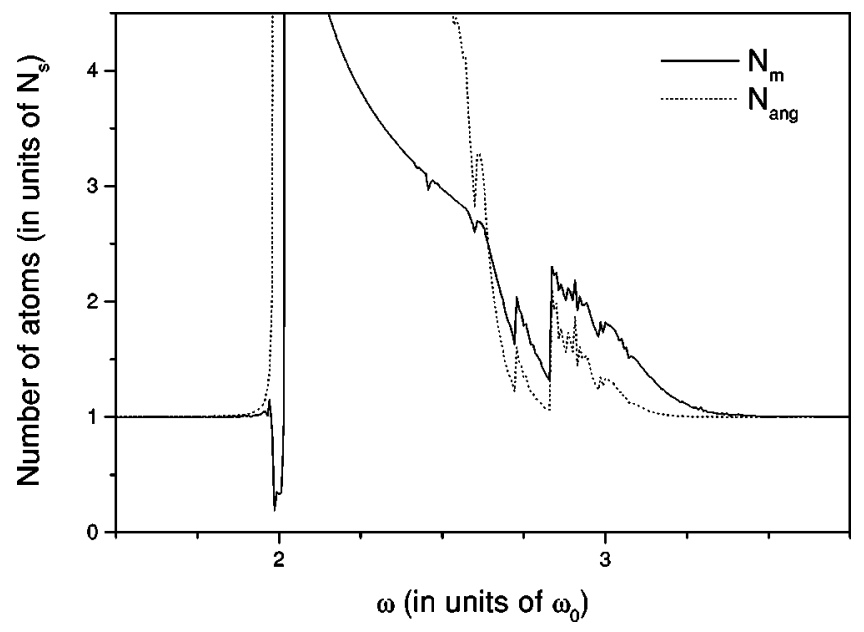

FIG. 1. Stabilization of an attractive condensate by weak driving as measured by the change in the maximum number of atoms participating in the condensate as a function of the driving frequency. The ratio $N_{\mathrm{m}}$ between the frequency-dependent maximum number of atoms $N_{\omega}$ and the maximum value $N_{\mathrm{s}}$ for the static condensate is shown. The figure is truncated at $N_{\mathrm{m}} \approx 4.8$ due to the excessive ( $t$ $>100 \mathrm{~s}$ ) amount of time taken to reach this value. An estimated value $N_{\text {ang }}$ for $N_{m}$ obtained from an angular momentum analysis is also shown. See text for details.

The normal modes of the quasiparticle dynamics around this minimum are the lowest-lying even collective excitations of the condensate $[10,14,15]$. These normal mode frequencies are obtained by computing the matrices $\mathcal{V}, T$ with elements $\mathcal{V}_{i j}=\partial^{2} H_{v a r} / \partial \rho_{i} \partial \rho_{j}, \mathcal{T}_{i j}=\partial^{2} H_{v a r} / \partial \Pi_{i} \partial \Pi_{j} \quad$ and then solving for the roots $\Omega_{i}$ of the equation $\operatorname{det}\left(\mathcal{V}-\Omega^{2} \mathcal{T}\right)$ $=0$. One solution is $\Omega_{1}=\sqrt{5-\rho_{\beta}^{-4}}$, which is the frequency for radial vibration of the quasiparticle or the condensate's "breathing' mode. The other two solutions are the degenerate solutions $\Omega_{2,3}=\sqrt{2+2 \rho_{\beta}^{-4}}$, where the vibrational motion is perpendicular to the radial direction. This can be visualized as being along the surface of a sphere of fixed radiusthese are the condensate's quadrupolar surface modes $[10,14,15]$. Since $\rho_{\beta}$ decreases as a function of $N_{0}, \Omega_{1}$ decreases and $\Omega_{2,3}$ increases with increasing $N_{0}$. Also note that $\Omega_{1,2,3}=\Omega_{0}=2$ for $\rho_{\beta}=1$, i.e. for $\beta=0$ (noninteracting limit) in these natural units.

We now turn to the numerical solution of this system of equations. As expected, these lowest-lying even collective modes of the condensate are excited by the driving. Driving the lowest such mode, a radial breathing mode, further destabilizes the condensate and decreases the maximum number of atoms below the static maximum $N_{\mathrm{s}}$. Remarkably, driving the next-highest excitations, which are quadrupolar surface modes, causes the condensate to become more stable. That is, for certain frequencies, atoms occupy the condensate in numbers significantly greater than $N_{\mathrm{s}}$ and the condensate is sustained for correspondingly longer times. We have studied in particular the frequency-dependent value of the maximum number of atoms $N_{\omega}$ that can be sustained in the presence of the driving. We find that this frequency response is broad and qualitatively robust, with similar features for a wide range of tested parameters and many alternative configurations for both the confining and perturbing fields. An example of these responses is shown in Fig. 1, along with a 
theoretical estimate $N_{\text {ang }}$ as explained below. In obtaining it, we use $\alpha_{3}=0.02=-2 \alpha_{1}=-2 \alpha_{2}$, which represents the effect of a Helmholtz field oriented along the axis of an IoffePritchard magnetic trap. The response is shown as the ratio $N_{m}=N_{\omega} / N_{\mathrm{s}}$. The broad stabilization effect is clear in this figure; further, it can be seen that the curve has some interesting structure, including in particular a signature dip corresponding to a parametric resonance between the radial and quadrupolar modes as explained below. The stabilization effect is so strong that in some cases it is no longer obvious whether and when a collapse occurs. It is possible for $N_{0}$ to grow so large that the loss rate equals the fill rate, even for relatively large $\rho$. The values in Fig. 1 are therefore calculated using a "worst case" definition of the collapse, as the $N_{0}$ value at which the inelastic decay terms in Eq. (4) exceed the growth rate $G_{0}$. Although it is unclear whether the physics of the true dynamical situation in these cases is fully captured by the simple kinetics of Eq. (4), the stabilization is nonetheless remarkable.

To understand the detailed structure of Fig. 1, consider the case of driving the condensate with $\omega<\Omega_{0}$. Note that the dynamics of the quasiparticle are always restricted to the $\rho_{1}=\rho_{2}$ plane by our choice of driving. At an arbitrary value of $\omega$, the driving is not initially resonant with either of the excitations and there is a negligible response from the condensate. As $N_{0}$ increases with time, however, $\Omega_{1}(\beta)$ will ultimately equal any $\omega<\Omega_{0}$. The breathing mode is excited as a result and the quasiparticle oscillates along the radial direction. As this oscillation increases in amplitude, the quasiparticle is driven into the attractive hole and the condensate collapses. The net effect is to decrease $N_{m}(\omega)$, with the impact being greatest for $\omega$ slightly less than $\Omega_{0}$. The more interesting case is for $\omega>\Omega_{0}$ where again, the driving is not initially resonant. As $N_{0}$ increases, however, the quadrupolar surface modes come into resonance. The quasiparticle then oscillates with increasingly greater amplitude in a direction perpendicular to the radial direction, still in the $\rho_{1}=\rho_{2}$ plane. An angular momentum vector $\vec{l}_{\hat{q}}(t)=\vec{\rho} \times \vec{\Pi}$ pointing in the $\hat{q}$ direction can be associated with this motion, where $\hat{q} \equiv(1 / \sqrt{2})(1,-1,0)$ is the normal to the $\rho_{1}=\rho_{2}$ plane. This angular momentum vector reverses orientation [16] during the oscillation, with its magnitude going to zero at the turning points of the oscillation. However, the mean-square value of this angular momentum increases as the surface mode acquires increasing energy. By virtue of the energy in this oscillation, the quasiparticle avoids the chasm of the attractive 'hole', and the condensate is stabilized, avoiding collapse even when the attractive interactions are significant. The above implies that the stabilizing effect may be quantitatively estimated by adding to the kinetic energy pressure terms an angular momentum term $\bar{l}^{2} /\left(2 m \rho^{2}\right)$ where $\bar{l}^{2}$ is the time average of $l_{\hat{q}}^{2}(t)$. This yields an estimated increase in the maximum number of atoms as $N_{\text {ang }} \equiv N_{m}(\bar{l} \neq 0) / N_{m}(l$ $=0)=\left(1+4 \bar{l}^{2} / 3\right)^{5 / 4}$; this estimate is plotted in Fig. 1 with $\overline{l^{2}}$ computed directly from the dynamics. It is clear from comparing the curves that this simple angular momentum argument captures the essential features of the stabilization. In

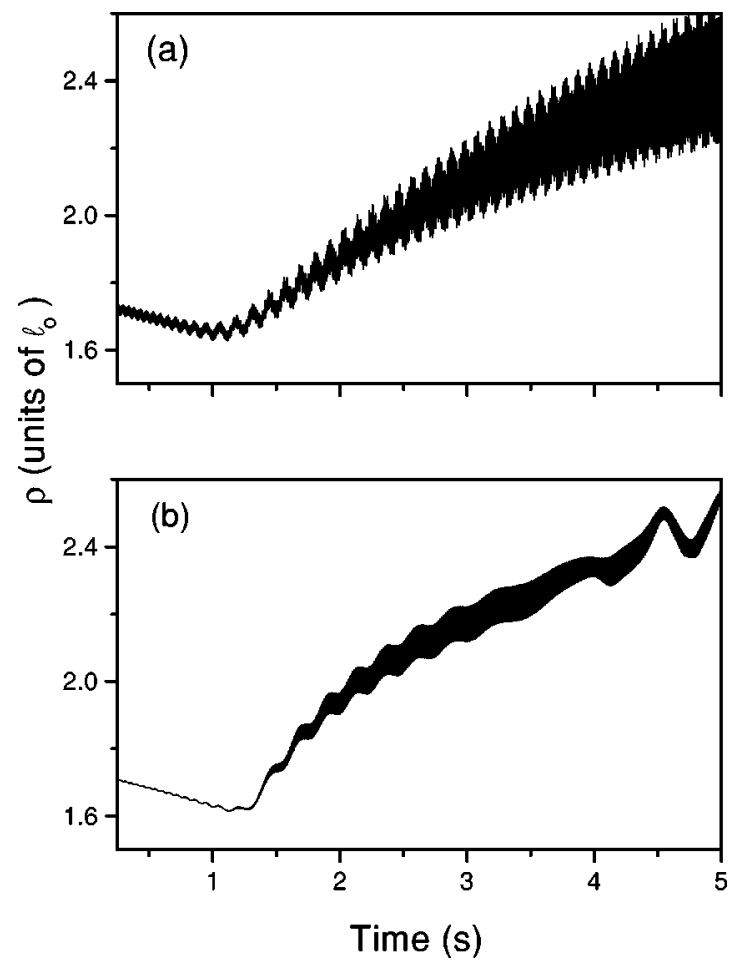

FIG. 2. (a) Time dependence of the radially averaged width of the condensate $\rho(t)$. There are rapid oscillations, as expected, on the time scale of the driving $(308 \mathrm{~Hz})$ that cannot be easily resolved on the scale of this figure. (b) As in (a) except as computed with a numerical solution of the GP equation with added dissipation. See text for details.

particular, the region of discrepancy is precisely where the naive kinetics and collapse criterion render suspect the value of $N_{\omega}$ shown, as argued above.

This resonant driving is easier to sustain for frequencies close to $\Omega_{0}$ since $\Omega_{2,3}$ has a weaker dependence on $N_{0}$ in that regime. As $N_{0}$ increases, the condensate ultimately falls out of resonance with the driving. Consider for example the condensate radius $\rho(t)=|\vec{\rho}(t)|$ : It is initially unaffected by the driving and decreases slowly as in the static case; this is followed by a resonantly driven increase that ultimately saturates. An example of this mechanism is shown in Fig. 2(a) for $\omega=2.3$ (corresponding to a driving frequency of $\approx 308 \mathrm{~Hz}$ for the Rice experiment). In Fig. 2(b) we show a numerical solution for the GP equation corresponding to the situation in Fig. 2(a); the two curves agree qualitatively. We note here that the numerical solution to the nonlinear Schrödinger equation in the GP form for cylindrical symmetry is extremely difficult and a full scan of frequencies is computationally prohibitive. However, simulations including filling and inelastic decay similar to those in the literature [8] have been performed to qualtitatively validate the GVA results.

As $\omega$ increases, the resonance occurs at increasingly later times and for decreasing windows of time, and hence the greatest stabilization happens for $\omega$ slightly greater than $\Omega_{0}$. There is no such stabilization for $\omega>\omega_{2}=\sqrt{12}=3.46$; this critical frequency is obtained by substituting the maximum value for the equilibrium $\rho_{m}$ in the expression for $\Omega_{2,3}-$ i.e., 
the driving cannot resonate with the condensate modes if the metastable minimum does not exist in the first place. This cutoff can be seen clearly in Fig. 1. Finally, we point out the significant dip in the curves at $\omega=\omega_{1}=\sqrt{8}$. This frequency corresponds to the situation where $\Omega_{2,3}(\beta)=2 \Omega_{1}(\beta)$, i.e., for $\rho_{\beta}^{-4}=3$. In this case, the driving and the surface modes parametrically excite the radial mode through the $1: 2$ resonance between the frequencies. Thus, the condensate is destabilized even for a frequency greater than $\Omega_{0}$. Similar resonances account for the other detailed structure in the frequency response curves. All these features should be clearly visible experimentally, since the experimental resolution is of the order of 50 condensate atoms [5], and the predicted features exceed this resolution significantly. Moreover, the details of the curves are sensitive to the precise models and parameters used. Comparison of experimental results with theory will therefore help improve our understanding of the rich interplay between kinetics and nonlinear dynamics in the attractive condensate. In particular, this provides a way to study the so far inaccessible collective modes of attractive condensates. This will open the way to further experiments and analyses and ultimately to a deeper understanding of this nonlinear many-body quantum regime. Efforts are under way for the experimental realization of these phenomena.

We gratefully acknowledge Keiko Petrosky and Hilary Lovett for their contributions to this research. A.K.P. benefited from useful discussions with Paul Stevenson. Research at Rice was supported by the NSF, ONR, the Welch Foundation and NASA. A.G. acknowledges partial support from the Fundação de Amparo à Pesquisa do Estado de São Paulo.
[1] M. H. Anderson et al., Science 269, 198 (1995); C. C. Bradley, C. A. Sackett, J. J. Tollett, and R. G. Hulet, Phys. Rev. Lett. 75, 1687 (1995); K. B. Davis et al., ibid. 75, 3969 (1995).

[2] F. Dalfovo, S. Giorgini, L. P. Pitaevskii, and S. Stringari, Rev. Mod. Phys. 71, 463 (1999), and references therein.

[3] See, for example, H. T. C. Stoof, Phys. Rev. A 49, 3824 (1994); N. Bogolubov, J. Phys. XI, 23 (1947).

[4] P. A. Ruprecht et al., Phys. Rev. A 51, 4704 (1995); C. C. Bradley, C. A. Sackett, and R. G. Hulet, Phys. Rev. Lett. 78, 985 (1997).

[5] C. A. Sackett, J. M. Gerton, M. Welling, and R. G. Hulet, Phys. Rev. Lett. 82, 876 (1999).

[6] J. M. Gerton, D. Strekalov, I. Prodan, and R. G. Hulet, Nature (London) 408, 692 (2000).

[7] C. A. Sackett, H. T. C. Stoof, and R. G. Hulet, Phys. Rev. Lett. 80, 2031 (1998).
[8] Yu. Kagan, A. E. Muryshev, and G. V. Shlyapnikov, Phys. Rev. Lett. 81, 933 (1998).

[9] S. L. Cornish et al., Phys. Rev. Lett. 85, 1795 (2000).

[10] V. M. Perez-Garcia et al., Phys. Rev. Lett. 77, 5320 (1996).

[11] A. K. Pattanayak and W. C. Schieve, Phys. Rev. E 50, 3601 (1994).

[12] The qualitative results have been verified to not depend on this assumption. The quantitative effects will be considered in detail elsewhere.

[13] J. M. Gerton, C. A. Sackett, B. J. Frew, and R. G. Hulet, Phys. Rev. A 59, 1514 (1999).

[14] K. Singh and D. Rokhsar, Phys. Rev. Lett. 77, 1667 (1996).

[15] M. Edwards et al., Phys. Rev. Lett. 77, 1671 (1996).

[16] Note that this is not a central force problem and as such the angular momentum is not conserved. 